\title{
Correction to: Assessment of tailings stability and soil contamination of Kef Ettout (NW Tunisia) abandoned mine
}

\author{
Nawrez Sliti ${ }^{1} \cdot$ Charef Abdelkrim $^{1} \cdot$ Lamia Ayed $^{2}$
}

Published online: 6 May 2019

(C) Saudi Society for Geosciences 2019

Correction to: Arabian Journal of Geosciences (2019) 12:73 https://doi.org/10.1007/s12517-018-4204-0

The original version of this paper was published with error. Tables and figures were renumbered during the publication process but captions retained as it was presented from original manuscript. Given in this article are the correct figures, tables and captions.

The online version of the original article can be found at https://doi.org/ $10.1007 / \mathrm{s} 12517-018-4204-0$

Charef Abdelkrim

Abdelkrim.Charef@ certe.rnrt.tn

1 Center of Water Researches and Technologies, BP 273, 8020 Soliman, Tunisia

2 Laboratoire d'Analyse, Traitement et Valorisation des Polluants de l'Environnement et des Produits, Faculté de Pharmacie de Monastir, Rue Avicenne, 5000 Monastir, Tunisia 


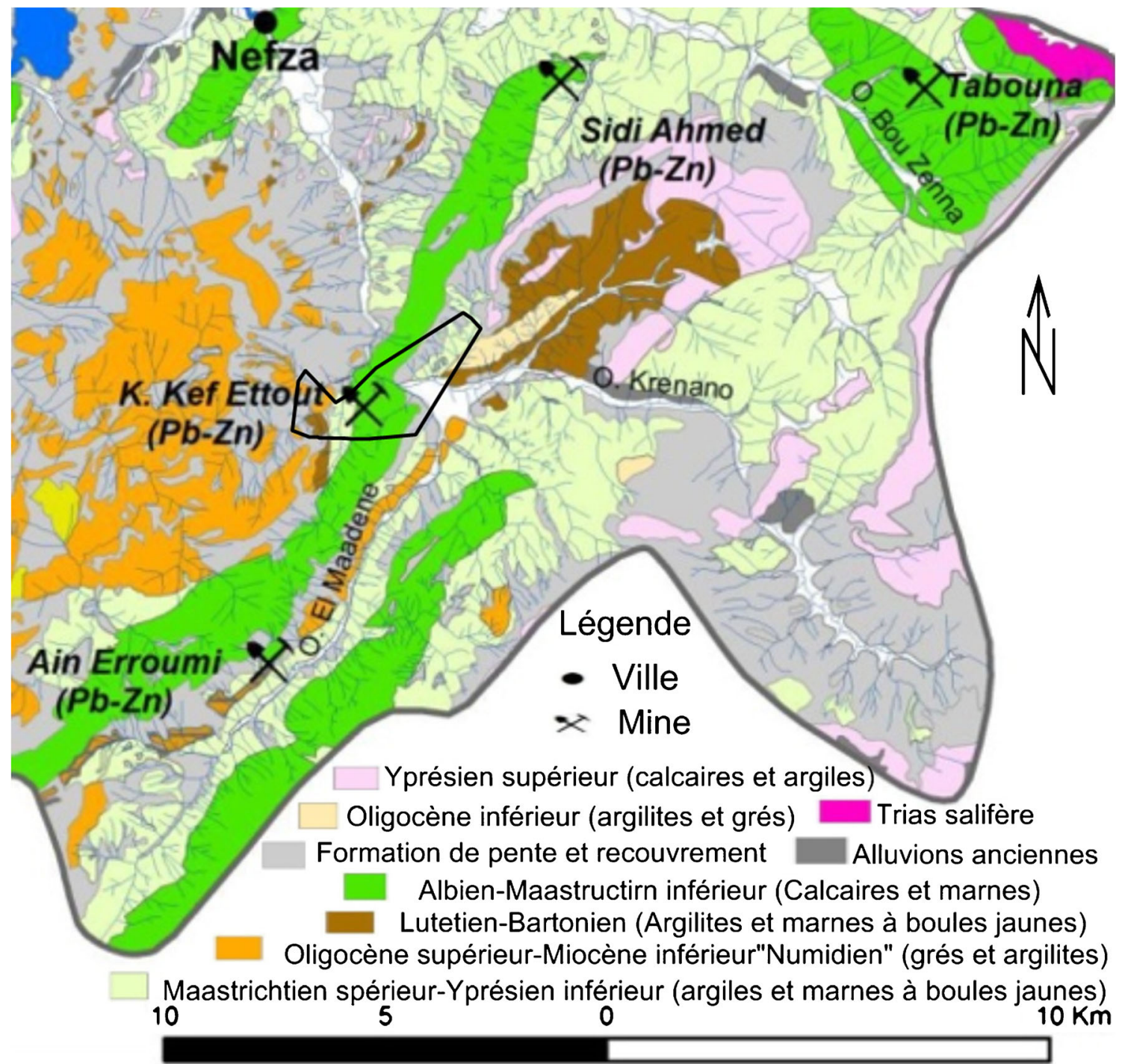

Fig. 1 Geological map of the of Wadi El Maadene catchment area and and location of study area (Rouvier, 1987; Batik, 1980) 


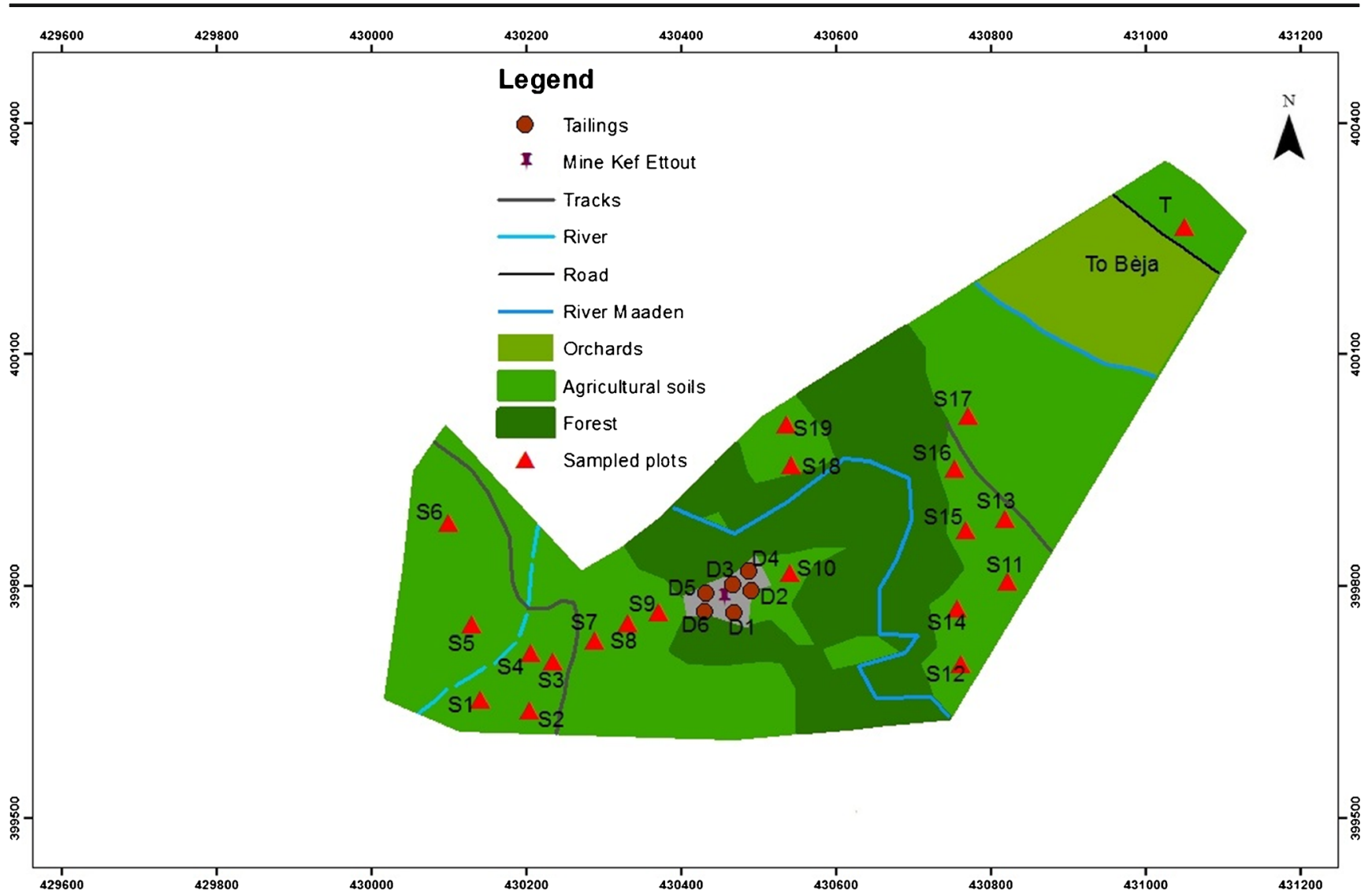

Fig. 2 Location of tailings and soils plots in the study area

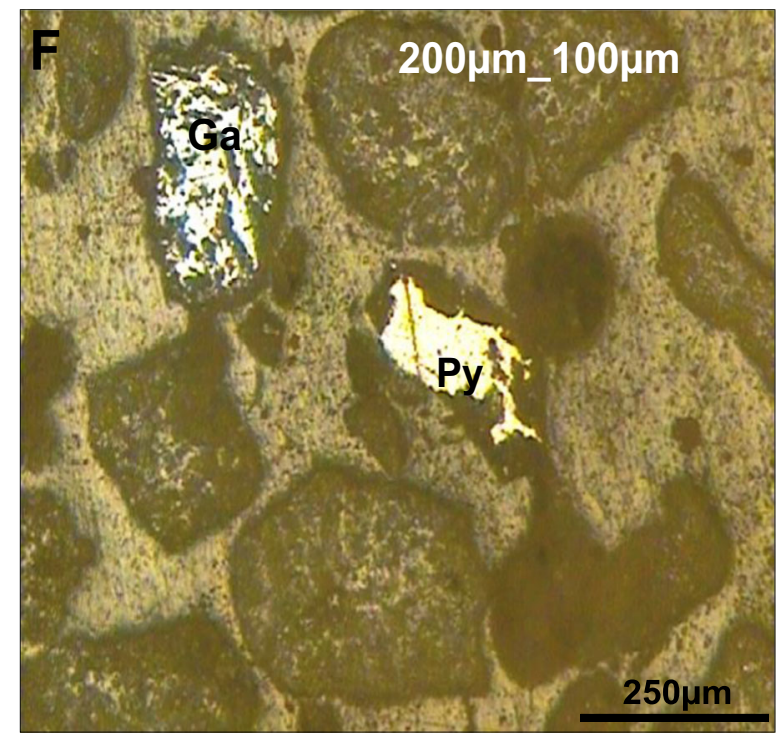

Photo 1 The progressed alteration from the external border towards the center of pyrite and galena 


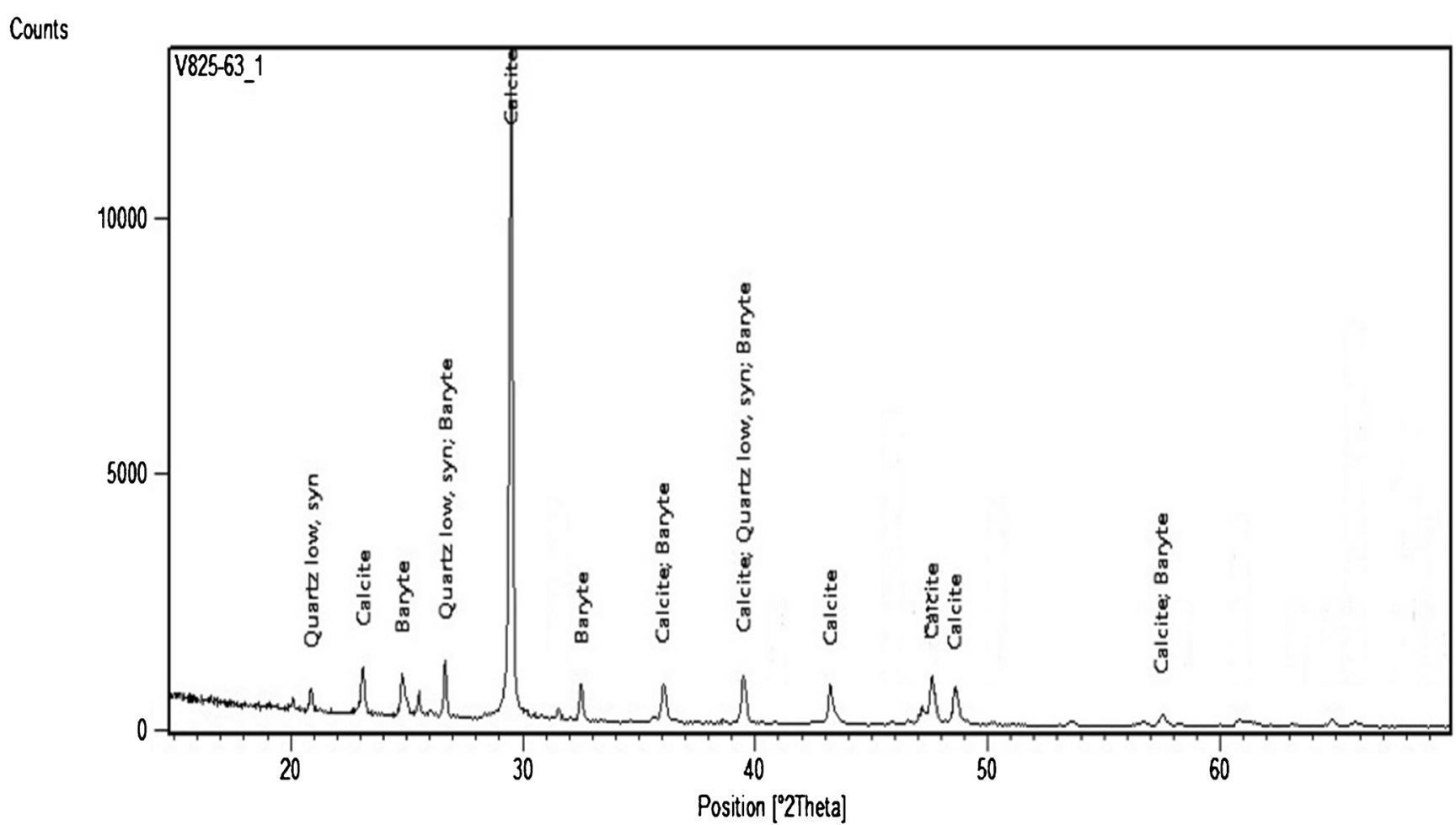

Fig. 3 RDX Diffractogramm of tailings sediments showing the presence of calcite, barite, quartz and essentially carbonates of the gangue and neoformed calcite 
Fig. 4 Spatial distribution of $\mathrm{Pb}$, $\mathrm{Zn}$ and $\mathrm{Cd}$ in study area
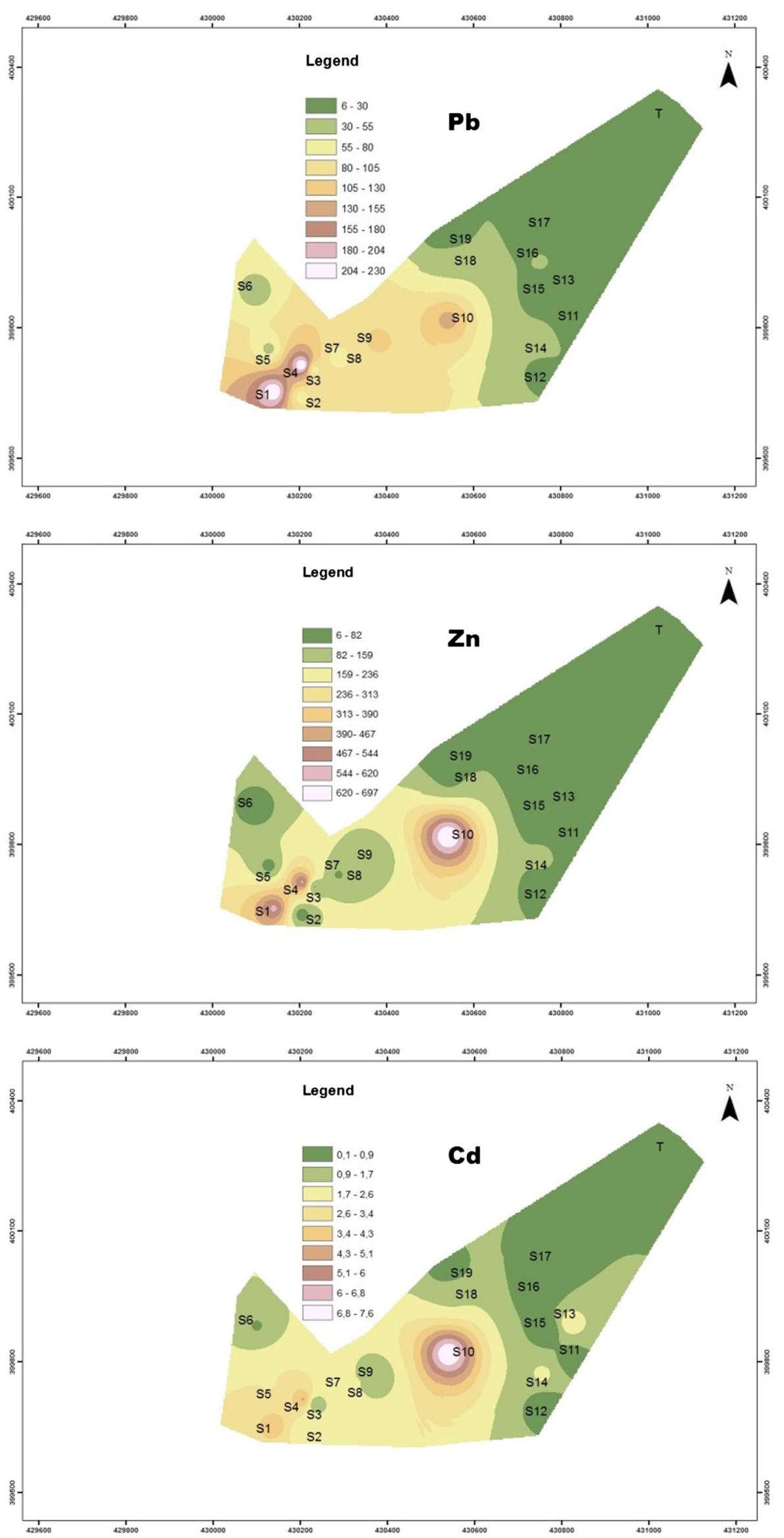

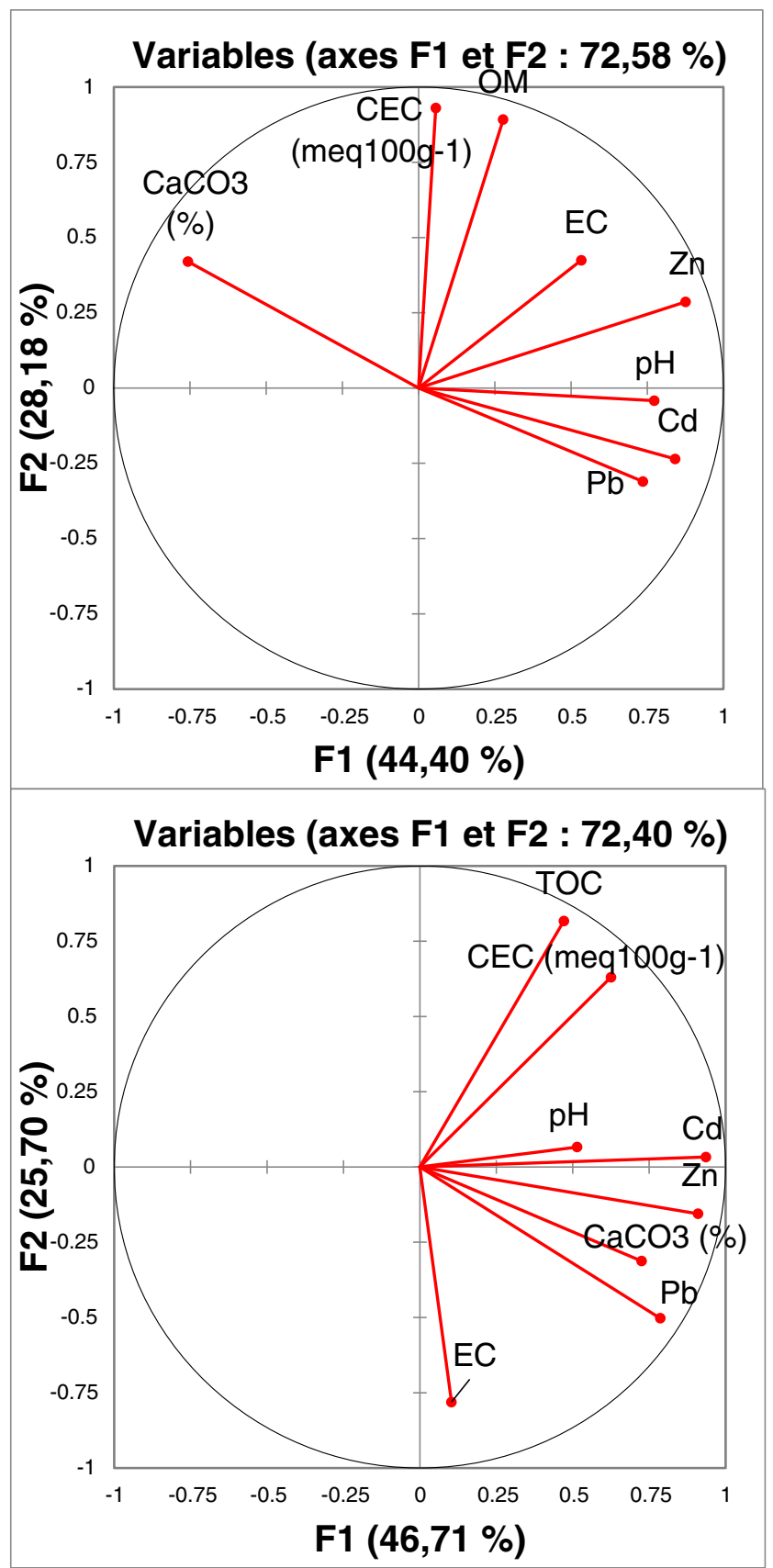

Fig. 5 Correlation circle on F1 and F2 of the tailings and agricultural soils

Fig. 6 Mean percentages of lead, zinc and cadmium bended to the four fractions of tailings sediments. Dn: Tailing number; F1: Exchangeable and carbonate bound Fraction); F2: Fe and Mn oxide bound Fraction; F3:

Organically and sulfur bound Fraction; F4: Residual Fraction

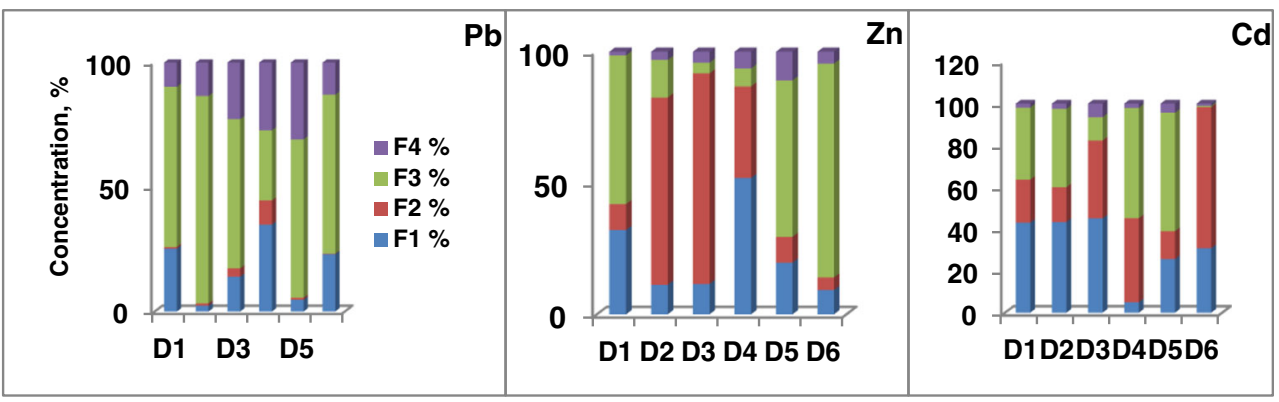




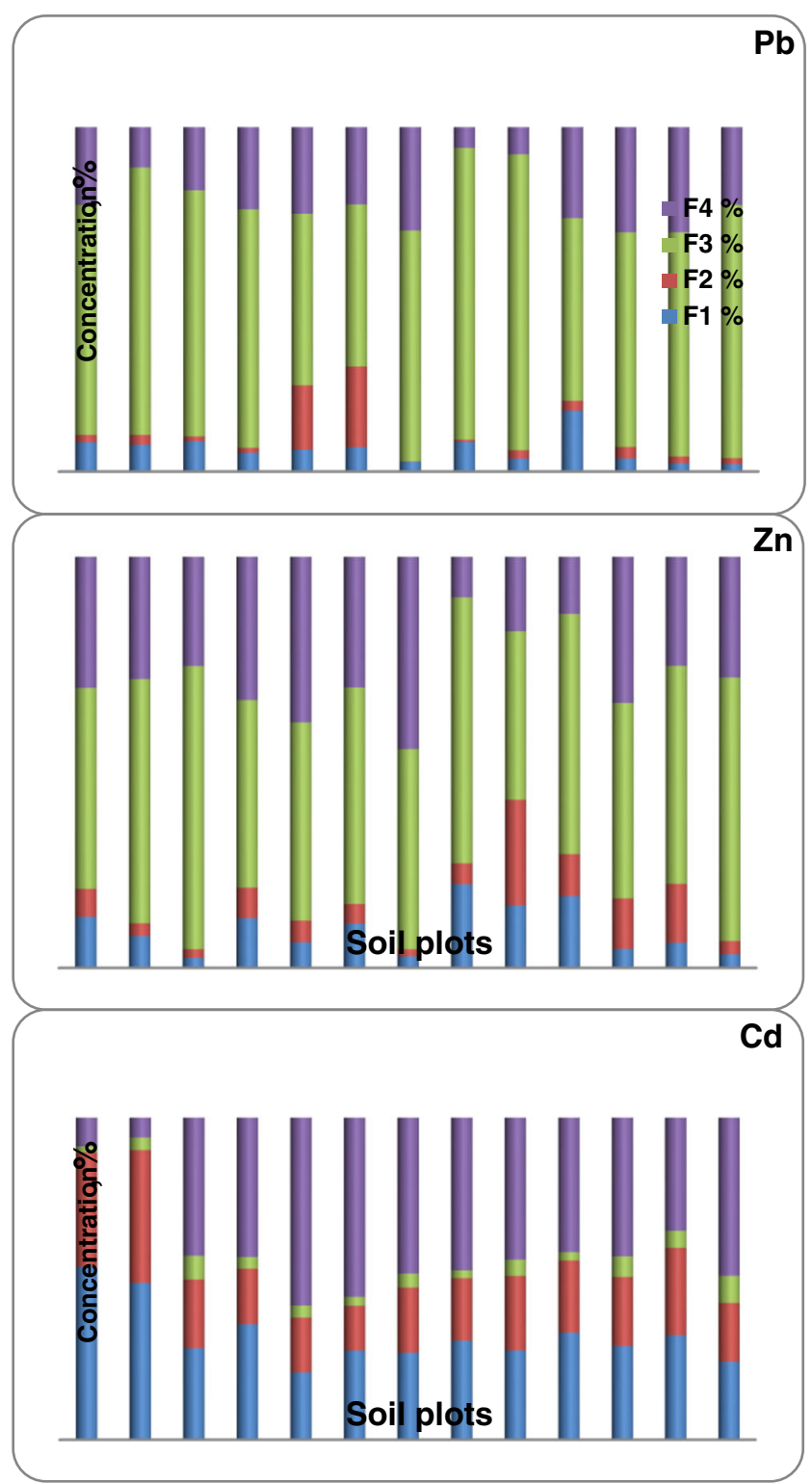

Fig. 7 Mean percentages of lead, zinc and cadmium bended to the four fractions of soil sediments. F1: Exchangeable and carbonate bound Fraction); F2: Fe and Mn oxide bound Fraction; F3: Organically and sulfur bound Fraction; F4: Residual Fraction

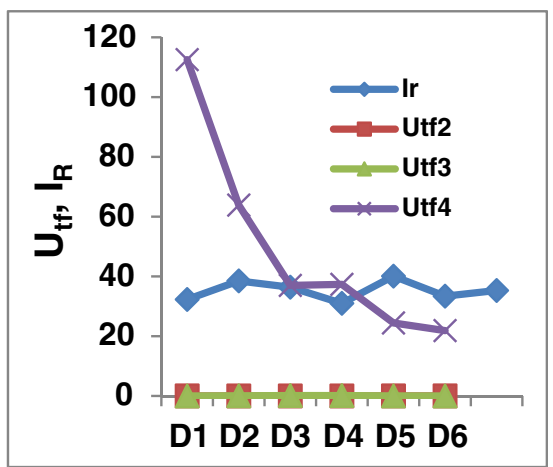

Fig. 8 Distribution indexes of exchangeable $\left(U_{t+1}\right)$, Fe-Mn oxides $\left(U_{t 22}\right)$ organic matter $\left(U_{\mathrm{tr} 3}\right)$ and residual $\left(\mathrm{U}_{\mathrm{tf} 4}\right)$ fractions and binding intensity $\left(I_{R}\right)$ of analyzed metals of tailings sediments. Dn: tailings number

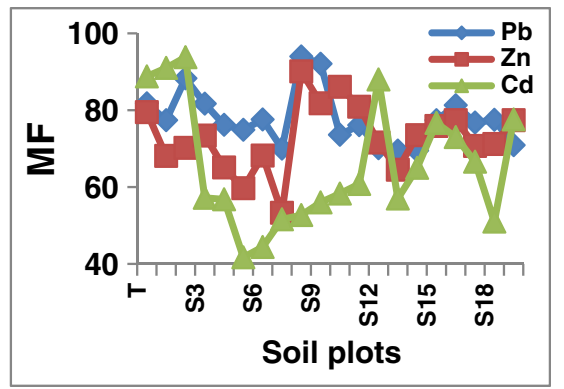

Fig. 9 Mobility factors (MF) of different metals percentages in control (T) and agricultural soils 
Table 1 Sequential extraction procedures BCR (the European Community Bureau of Reference)

\begin{tabular}{|c|c|c|c|}
\hline Extraction methods & Chemical composition & References & \\
\hline \multirow[t]{4}{*}{ Sequential extraction } & $\begin{array}{l}\text { F1 (Exchangeable and carbonate } \\
\text { bound Fraction): }\end{array}$ & $0.11 \mathrm{molL}^{-1}$ of $\mathrm{AcOH}$ & \multirow{4}{*}{$\begin{array}{l}\text { Davidson et al., 1994; } \\
\text { Ure et al., 1993; } \\
\text { Davidson et al., 1998; } \\
\text { Rauret et al., } 2000\end{array}$} \\
\hline & F2 (Fe and Mn oxide bound Fraction) & $\mathrm{NH}_{2} \mathrm{OH}-\mathrm{HCl}\left(0.1 \mathrm{molL}^{-1}, \mathrm{pH} 2\right)$ & \\
\hline & F3 (Organically and sulfur bound Fraction.) & $\begin{array}{l}\mathrm{H}_{2} \mathrm{O}_{2}\left(8.8 \mathrm{molL}^{-1}, \mathrm{pH} 3.2\right) \text { and } \mathrm{AcONH}_{4} \\
\quad\left(1 \mathrm{molL}^{-1}, \mathrm{pH} 2\right)\end{array}$ & \\
\hline & F4 (Residual Fraction) & Digestion $\left(\mathrm{HCl}-\mathrm{HNO}_{3}\right)+\mathrm{HF}\left(150^{\circ} \mathrm{C}-2 \mathrm{~h}\right)$ & \\
\hline
\end{tabular}

Table 2 Mean values of physicochemical proprieties of the tailings plots

\begin{tabular}{llllllllll}
\hline & $\mathrm{pH}$ & $\begin{array}{l}\mathrm{EC} \\
\left(\mathrm{mScm}^{-1}\right)\end{array}$ & $\begin{array}{l}\mathrm{CaCO}_{3} \\
(\%)\end{array}$ & $\begin{array}{l}\mathrm{OM} \\
(\%)\end{array}$ & $\begin{array}{l}\mathrm{CEC} \\
\left(\mathrm{meq} 100 \mathrm{~g}^{-1}\right)\end{array}$ & $\begin{array}{l}\text { Sand } \\
(\%)\end{array}$ & $\begin{array}{l}\text { Clay } \\
(\%)\end{array}$ & $\begin{array}{l}\text { Silt } \\
(\%)\end{array}$ & Texture \\
\hline $\mathrm{D} 1$ & 7.8 & 500 & 74 & 0.7 & 8 & 53 & 6 & 41 & Sandy-loam \\
$\mathrm{D} 2$ & 8 & 580 & 78 & 1.4 & 13 & 70 & 8 & 22 & Sandy-loam \\
D3 & 7.5 & 570 & 59 & 0.5 & 9 & 51 & 3 & 46 & Sandy-loam \\
D4 & 7.1 & 560 & 59 & 1.3 & 12 & 32 & 18 & 50 & Loamy-sand \\
D5 & 7.7 & 570 & 62 & 1.0 & 8 & 53 & 8 & 39 & Sandy-loam \\
D6 & 8.2 & 550 & 58 & 1.2 & 12 & 24 & 16 & 60 & Sandy-loam \\
\hline
\end{tabular}

D: tailings; TOC: Total organic carbon; EC: Electric conductivity; CEC: Cationexchange capacity; OM: Organic matter

Table 3 Physicochemical proprieties of initial wastes of ore valorization unity (WOVU)

\begin{tabular}{lllllllll}
\hline & $\mathrm{pH}$ & $\begin{array}{l}\mathrm{EC} \\
\left(\mathrm{mScm}^{-1}\right)\end{array}$ & $\begin{array}{l}\mathrm{CaCO}_{3} \\
(\%)\end{array}$ & $\begin{array}{l}\mathrm{TOC} \\
\left(\mathrm{gkg}^{-1}\right)\end{array}$ & $\begin{array}{l}\mathrm{CEC} \\
\left(\mathrm{meq} 100 \mathrm{~g}^{-1}\right)\end{array}$ & $\begin{array}{l}\text { Sand } \\
(\%)\end{array}$ & $\begin{array}{l}\text { Clay } \\
(\%)\end{array}$ & $\begin{array}{l}\text { Silt } \\
(\%)\end{array}$ \\
\hline Min & 6.5 & 621.2 & 42.3 & 2.1 & 15.9 & 28.9 & 21.6 & 44.9 \\
Max & 6.9 & 703.2 & 51.6 & 4.2 & 21.3 & 32.6 & 24.2 & 55.6 \\
Mean & 6.8 & 661.9 & 47.0 & 2.9 & 17.9 & 30.8 & 19.1 & 50.1 \\
$\mathrm{SD}$ & 0,2 & 38.7 & 5.1 & 0.9 & 3.1 & 2.1 & 1.4 & 6.3 \\
$\mathrm{~N}$ & 69 & 69 & 69 & 69 & 69 & & & \\
\hline
\end{tabular}

N: Samples number, Max: Maximum; Min: Minimum; SD:Standard deviation; TOC: Total organic carbon 
Table 4 Mean values of physicochemical proprieties of the soil plots

\begin{tabular}{llllllllll}
\hline & $\mathrm{pH}$ & $\begin{array}{l}\mathrm{EC} \\
\left(\mathrm{mScm}^{-1}\right)\end{array}$ & $\begin{array}{l}\mathrm{CaCO}_{3} \\
(\%)\end{array}$ & $\begin{array}{l}\mathrm{CEC} \\
\left(\mathrm{meq} 100 \mathrm{~g}^{-1}\right)\end{array}$ & $\begin{array}{l}\mathrm{TOC} \\
(\%)\end{array}$ & $\begin{array}{l}\text { Sand } \\
(\%)\end{array}$ & $\begin{array}{l}\text { Clay } \\
(\%)\end{array}$ & $\begin{array}{l}\text { Silt } \\
(7)\end{array}$ \\
\hline $\mathrm{T}$ & 7 & 400 & 19 & 17 & 0.75 & 7 & 43 & 50 & Silty-clay \\
$\mathrm{S} 1$ & 7.7 & 460 & 21 & 19 & 1.01 & 40 & 51 & $\mathrm{~S} 1$ & Silty-clay \\
$\mathrm{S} 2$ & 7.5 & 500 & 17 & 17 & 1.27 & 19 & 65 & $\mathrm{~S} 2$ & Silty \\
$\mathrm{S} 3$ & 7.7 & 530 & 24 & 17 & 1.28 & 20 & 55 & $\mathrm{~S} 3$ & Silty \\
$\mathrm{S} 4$ & 7.2 & 520 & 22 & 18 & 0.45 & 17 & 73 & $\mathrm{~S} 4$ & Silty \\
$\mathrm{S} 5$ & 7.4 & 530 & 18 & 21 & 1.67 & 45 & 43 & $\mathrm{~S} 5$ & Silty-clay \\
$\mathrm{S} 6$ & 8 & 480 & 19 & 17 & 1.43 & 19 & 65 & $\mathrm{~S} 6$ & Silty \\
$\mathrm{S} 7$ & 7.1 & 480 & 23 & 19 & 1.8 & 46 & 42 & $\mathrm{~S} 7$ & clay-silty \\
$\mathrm{S} 8$ & 8.1 & 490 & 24 & 17 & 1.35 & 32 & 50 & $\mathrm{~S} 8$ & Silty-clay \\
$\mathrm{S} 9$ & 7.9 & 420 & 22 & 19 & 1.93 & 41 & 42 & $\mathrm{~S} 9$ & Silty-clay \\
$\mathrm{S} 10$ & 8 & 400 & 25 & 26 & 4.5 & 59 & 32 & $\mathrm{~S} 10$ & Clay-loam \\
$\mathrm{S} 11$ & 7.5 & 450 & 16 & 17 & 1.81 & 30 & 55 & $\mathrm{~S} 11$ & Silty-clay \\
$\mathrm{S} 12$ & 7.7 & 420 & 18 & 21 & 2.25 & 50 & 42 & $\mathrm{~S} 12$ & Clay-loam \\
$\mathrm{S} 13$ & 7.4 & 410 & 16 & 24 & 2.51 & 55 & 35 & $\mathrm{~S} 13$ & Clay-loam \\
$\mathrm{S} 14$ & 7.6 & 420 & 20 & 16 & 1.6 & 36 & 40 & $\mathrm{~S} 14$ & Silty-clay \\
$\mathrm{S} 15$ & 7.3 & 440 & 16 & 16 & 1.51 & 34 & 38 & $\mathrm{~S} 15$ & Silty-clay \\
$\mathrm{S} 16$ & 7.4 & 430 & 19 & 17 & 1.72 & 41 & 46 & $\mathrm{~S} 16$ & Silty-clay \\
$\mathrm{S} 17$ & 7.2 & 420 & 16 & 16 & 1.51 & 34 & 39 & $\mathrm{~S} 17$ & Silty-clay \\
$\mathrm{S} 18$ & 7.5 & 400 & 20 & 21 & 1.75 & 43 & 50 & $\mathrm{~S} 18$ & Silty-clay \\
$\mathrm{S} 19$ & 7.3 & 420 & 15 & 19 & 2.03 & 35 & 52 & $\mathrm{~S} 19$ & Silty-clay \\
\hline
\end{tabular}

TOC: total organic carbon; T: Control soil: S: soil plot
Table $5 \mathrm{~Pb}, \mathrm{Zn}$ and $\mathrm{Cd}$ means concentrations, standard deviations, maximum and minimum concentrations in initial wastes of ore valorization unity (WOVU) in $\mathrm{mgkg}^{-1}$

\begin{tabular}{llll}
\hline & $\mathrm{Pb}$ & $\mathrm{Zn}$ & $\mathrm{Cd}$ \\
\hline Min & $14,177.00$ & $22,124.00$ & 1649.00 \\
Max & $15,277.00$ & $24,591.00$ & 2213.00 \\
Mean & $14,505.00$ & $23,177.00$ & 1908.00 \\
$\mathrm{SD}$ & 751.00 & 1391.00 & 271.30 \\
\hline
\end{tabular}

SD: standard deviations, Maximum: Max;Minimum: Min
Table 6 Mean concentrations and their standards Deviations of potentially toxic metals of tailings plots $\left(\mathrm{mgkg}^{-1}\right)$

\begin{tabular}{lllllll}
\hline Plot tailings & $\mathrm{Pb}$ & $\mathrm{SD}$ & $\mathrm{Zn}$ & $\mathrm{SD}$ & $\mathrm{Cd}$ & $\mathrm{SD}$ \\
\hline $\mathrm{D} 1$ & 3250.00 & 861.00 & 2165.00 & 787.00 & 18.00 & 1.7 \\
D2 & 3281.00 & 120.00 & 4900.00 & 672.00 & 18.00 & 2.0 \\
D3 & 8984.00 & 1265.00 & 5330.00 & 1072.00 & 69.00 & 4.6 \\
D4 & $11,082.00$ & 2268.00 & $15,000.00$ & 2123.00 & 77.50 & 7.8 \\
D5 & 3486.00 & 266.00 & 4900.00 & 316.00 & 12.50 & 1.3 \\
D6 & $37,400.00$ & 3435.00 & 9520.00 & 729.00 & 90.00 & 2.7 \\
\hline
\end{tabular}

Dx: talings number; SD: Standard deviation 
Table 7 Mean concentrations and their standard deviations of potentially toxic metals of soil plots $\left(\mathrm{mgkg}^{-1}\right)$

\begin{tabular}{lllllll}
\hline & $\mathrm{Pb}$ & $\mathrm{SD}$ & $\mathrm{Zn}$ & $\mathrm{SD}$ & $\mathrm{Cd}$ & $\mathrm{SD}$ \\
\hline $\mathrm{T}$ & 10 & 4 & 7 & 3 & 0.1 & 0.0 \\
$\mathrm{~S} 1$ & 227 & 28 & 556 & 49 & 4.0 & 0.8 \\
$\mathrm{~S} 2$ & 70 & 14 & 44 & 4 & 2.0 & 0.9 \\
$\mathrm{~S} 3$ & 72 & 10 & 80 & 10 & 1.0 & 0.3 \\
$\mathrm{~S} 4$ & 230 & 29 & 551 & 32 & 4.4 & 1.1 \\
S5 & 49 & 8 & 60 & 8 & 2.6 & 0.2 \\
S6 & 44 & 6 & 41 & 8 & 0.9 & 0.3 \\
S7 & 67 & 6 & 73 & 17 & 2.2 & 0.3 \\
S8 & 82 & 7 & 151 & 10 & 1.8 & 0.3 \\
S9 & 114 & 7 & 99 & 9 & 1.0 & 0.2 \\
S10 & 138 & 8 & 698 & 17 & 7.7 & 0.4 \\
S11 & 14 & 5 & 42 & 5 & 0.4 & 0.1 \\
S12 & 24 & 5 & 45 & 8 & 0.3 & 0.1 \\
S13 & 22 & 7 & 32 & 7 & 2.5 & 0.2 \\
S14 & 44 & 6 & 98 & 9 & 2.0 & 0.3 \\
S15 & 16 & 3 & 9 & 4 & 0.1 & 0.0 \\
S16 & 33 & 4 & 23 & 7 & 0.2 & 0.1 \\
S17 & 7 & 3 & 6 & 3 & 0.2 & 0.1 \\
S18 & 53 & 8 & 62 & 8 & 1.6 & 0.3 \\
S19 & 6 & 4 & 16 & 4 & 0.3 & 0.2 \\
\hline
\end{tabular}

T: Control soil; SD: Standard deviation
Table 8 Comparison of potentially toxic metal contents with some background values $\left(\mathrm{mgkg}^{-1}\right)$

\begin{tabular}{|c|c|c|c|c|c|c|c|}
\hline & \multirow{2}{*}{ This study } & \multirow{2}{*}{ Control soil } & \multicolumn{5}{|c|}{ Reference values } \\
\hline & & & 1 & 2 & 3 & 4 & 5 \\
\hline $\mathrm{Pb}$ & $6-230$ & 6 & 50 & $9-50$ & 25 & 10 & 35 \\
\hline $\mathrm{Zn}$ & $6-698$ & 7 & 100 & $10-100$ & 62 & & 90 \\
\hline $\mathrm{Cd}$ & $0.1-7.77$ & 0.1 & 3 & $0.05-0.45$ & - & 0.3 & 0.35 \\
\hline \multicolumn{8}{|c|}{$\begin{array}{l}\text { 1: Canadian Canadian Norms of the total contents of the ETM (mgkg- } \\
\text { 1) in soils (the Canadian Council of Ministers of the environment,1991) } \\
\text {; 2: Ordinary soils (Baize, 1997) ; 3: Worldwide data (Kabata-Pendias } \\
\text { and Pendias, 1999); 4: WHO (cited by Parizanganeh et al., 2012); 5: } \\
\text { Bowen (1979) }\end{array}$} \\
\hline
\end{tabular}

